IMMUNOLOGY

\title{
Immune topology of the human kidney
}

A variety of immune cells exist within the human kidney; however, the exact cell types, their spatiotemporal arrangements and their cell-signalling circuitry are not well defined. Using single-cell RNA sequencing (scRNA-seq) and mass cytometry analyses, researchers have now produced a topology of immune cells within the human kidney, which describes how immune cell populations change over time and within different anatomical zones in response to cell-derived signals. "Surprisingly, we found that the kidney contains a varied network of tissue-resident myeloid and lymphoid cells; these are seeded during development but their functionality changes over time, with postnatal acquistion of gene expression programmes that promote defence against infection," says Menna Clatworthy. "We also reveal that epithelial cells in different anatomical regions express different immune genes to orchestrate the localization of immune cells to specific regions, and that this capability is not present in fetal kidneys."

Organ-resident immune cells are

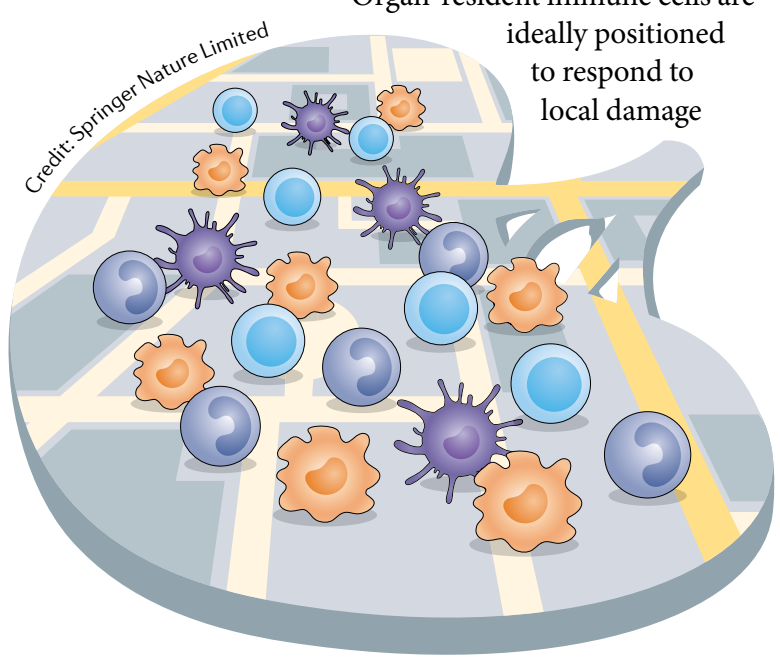

or infection and may determine whether these insults are resolved or progress. In previous work, Clatworthy and colleagues demonstrated that the high interstitial sodium concentration of the medulla stimulates the production of chemokines by tubular epithelial cells, which helps position macrophages to counter the immunological threat posed by ascending bacterial infection. As Clatworthy explains, several questions arose from this work. "We wanted to know whether and where other immune cell subsets reside in the kidney, and whether kidney epithelial cells have other immune functions beyond chemokine production," she says. "We also wanted to know whether the immune cells in the human kidney are seeded in the developing fetal kidney or if they migrate there after birth, and how they change with age."

To assess the immune landscape of the human kidney, the researchers performed scRNA-seq on 14 mature kidney samples and 6 fetal kidney samples, profiling the RNA transcripts in a total of 40,268 mature and 27,203 fetal kidney cells. Cells were grouped on the basis of their gene expression profiles to generate clusters of immune and non-immune cells and produce a global 'atlas' of cells in the kidney. "We supported this dataset with flow and mass cytometry experiments to validate the immune cell populations identified, and examined the functional consequences of the spatial gene expression patterns in a mouse model of pyelonephritis," explains Clatworthy.

The researchers show that a number of immune cell populations are established in the human kidney in the first trimester of development but that the transcriptional profiles of these cells differ from those of immune cells in the mature kidney, with postnatal acquisition of transcriptional signatures indicative of roles in inflammation and immune defence. The findings also support a role for crosstalk between resident epithelial cells and immune cells in determining the spatiotemporal zonation of immune cells. In contrast to the epithelium of human fetal kidney, which showed little expression of immune genes, epithelial cells of the mature kidney - particularly those of the kidney pelvis - showed higher expression of genes involved in bacterial defence, such as TLR5 and MYD88. Analysis of chemokineligand interactions showed expression of chemokine ligands on epithelial cells of the connecting nephron and kidney pelvis with expression of their corresponding receptors by macrophages and neutrophils. The functional relevance of these interactions was demonstrated in a mouse model of pyelonephritis, in which the expression of chemokines in the medulla and pelvis were increased following bacterial challenge, resulting in an accumulation of neutrophils in the renal pelvis. Of note, the pelvic epithelium of human kidney samples also showed high expression of antimicrobial peptides, suggesting that epithelial and immune cells contribute to antimicrobial defence.

The researchers say that their atlas of cell types and gene expression in the human adult and fetal kidney will be freely available via the Human Cell Atlas project and the Kidney Cell Atlas. "This map will transform our understanding of cellular networks in the kidney," says Clatworthy. "Future work will aim to understand the ontogeny, localization and functions of resident immune cells, both in kidney physiology and in disease."

Susan J. Allison

ORIGINAL ARTICLE Stewart, B. J. et al.

Spatiotemporal immune zonation of the human kidney. Science 365, 1461-1466 (2019) RELATED LINK Kidney Cell Atlas: https://www. kidneycellatlas.org/ 